\title{
Eccentric exercise and delayed onset muscle soreness of the quadriceps induce adjustments in agonist-antagonist activity, which are dependent on the motor task
}

\author{
C. Vila-Chã $\cdot$ H. Hassanlouei • D. Farina \\ D. Falla
}

Received: 29 June 2011 / Accepted: 4 November 2011/Published online: 18 November 2011

(C) The Author(s) 2011. This article is published with open access at Springerlink.com

\begin{abstract}
This study investigates the effects of eccentric exercise and delayed onset muscle soreness (DOMS) of the quadriceps on agonist-antagonist activity during a range of motor tasks. Ten healthy volunteers (age, mean $\pm \mathrm{SD}$, $24.9 \pm 3.2$ years) performed maximum voluntary contractions (MVC) and explosive isometric contractions of the knee extensors followed by isometric contractions at $2.5,5,10,15,20$, and $30 \%$ MVC at baseline, immediately after and $24 \mathrm{~h}$ after eccentric exercise of the quadriceps. During each task, force of the knee extensors and surface EMG of the vasti and hamstrings muscles were recorded concurrently. Rate of force development (RFD) was computed from the explosive isometric contraction, and the coefficient of variation of the force $(\mathrm{CoV})$ signal was estimated from the submaximal contractions. Twenty-four hours after exercise, the subjects rated their perceived pain intensity as $4.1 \pm 1.2$ (score out of 10). The maximum
\end{abstract}

C. Vila-Chã

Polytechnic Institute of Bragança, Bragança, Portugal

H. Hassanlouei

Center for Sensory-Motor Interaction, Department of Health

Science and Technology, Aalborg University, Aalborg, Denmark

D. Farina $\cdot$ D. Falla $(\bowtie)$

Department of Neurorehabilitation Engineering, Bernstein Focus Neurotechnology Göttingen, Bernstein Center for Computational Neuroscience, University Medical Center Göttingen,

Georg-August University, Von-Siebold-Str. 4,

37075 Göttingen, Germany

e-mail: deborah.falla@bccn.uni-goettingen.de

D. Falla

Pain Clinic, Center for Anesthesiology, Emergency and Intensive Care Medicine, University Hospital Göttingen, Göttingen, Germany
RFD and MVC of the knee extensors was reduced immediately post- and $24 \mathrm{~h}$ after eccentric exercise compared to baseline (average across both time points: $19.1 \pm 17.1 \%$ and $11.9 \pm 9.8 \%$ lower, respectively, $P<0.05)$. The $\mathrm{CoV}$ for force during the submaximal contractions was greater immediately after eccentric exercise (up to 66\% higher than baseline, $P<0.001$ ) and remained higher $24 \mathrm{~h}$ postexercise during the presence of DOMS $(P<0.01)$. For the explosive and MVC tasks, the EMG amplitude of the vasti muscles decreased immediately after exercise and was accompanied by increased antagonist EMG for the explosive contraction only. On the contrary, reduced force steadiness was accompanied by a general increase in EMG amplitude of the vasti muscles and was accompanied by increased antagonist activity, but only at higher force levels ( $>15 \%$ MVC). This study shows that eccentric exercise and subsequent DOMS of the quadriceps reduce the maximal force, rate of force development and force steadiness of the knee extensors, and is accompanied by different adjustments of agonist and antagonist muscle activities.

Keywords Delayed onset muscle soreness . Eccentric exercise $\cdot$ Muscle damage

\section{Introduction}

The neuromuscular system produces an extensive repertoire of force and movement, which ranges from precise movements to powerful movements involving the maximum capacity of the muscle to generate force at maximum velocity (Bawa 2002). Depending on the characteristics of the motor task, multiple features of motor control determine the degree of muscle activation and consequently the magnitude, precision, and speed of force output (Enoka 
1988; Bawa 2002; Taylor et al. 2003). For instance, the rate of force development is influenced by the ability of the nervous system to recruit motor units at higher frequencies than needed to achieve full tetanic fusion (Desmedt and Godaux 1977), while the maintenance of stable contractions is more affected by motor unit discharge rate variability (Tracy et al. 2005).

Motor output can be impaired by unaccustomed exercise, particularly by eccentric exercise (Sargeant and Dolan 1987; Saxton et al. 1995; Crameri et al. 2007; Semmler et al. 2007; Meszaros et al. 2010). Maximal force (Prasartwuth et al. 2005; Crameri et al. 2007), power (Sargeant and Dolan 1987; Crameri et al. 2007), and force steadiness (Saxton et al. 1995; Semmler et al. 2007; Meszaros et al. 2010) are reduced following intensive eccentric exercise. These alterations have commonly been associated to morphological alterations of the muscle, such as myofibrillar disruption, disturbance of the extracellular matrix, and an inflammatory reaction, which results in delayed onset muscle soreness (DOMS) and muscle stiffness (Jones et al. 1987; Howell et al. 1993; Yu and Thornell 2002).

The extent to which eccentric exercise and exerciseinduced DOMS affects a motor task may vary depending on the characteristics of the task; for example, the rate of force development is more influenced than maximal force by eccentric exercise (Crameri et al. 2007), and during constant force tasks, the force variability increases more at very low force levels ( 2.5 and $5 \% \mathrm{MVC})$ than at moderate force levels (10-30\% MVC) (Dartnall et al. 2008). Since different force tasks involve different motor control strategies, the degree of impairment might be determined by changes within the nervous system, which ultimately affect the neural activation of agonist and antagonist muscles (Semmler et al. 2007; Dartnall et al. 2008, 2009; Meszaros et al. 2010; Piitulainen et al. 2010). For example, during maximal isometric contractions, the activity of agonist muscles is depressed for $2 \mathrm{~h}$ after eccentric exercise, but recovers following a period of $24 \mathrm{~h}$ (Meszaros et al. 2010; Piitulainen et al. 2010), while at submaximal force levels, the agonist and antagonist muscle activities are increased (Semmler et al. 2007; Dartnall et al. 2008, 2009). Nonetheless the results are inconsistent (Leger and Milner 2001; Prasartwuth et al. 2005; Semmler et al. 2007; Paschalis et al. 2007; Meszaros et al. 2010; Piitulainen et al. 2010). For instance, in the $2 \mathrm{~h}$ following eccentric exercise of the elbow flexors, the biceps brachii EMG has been shown to decrease (Piitulainen et al. 2010) or to remain unchanged (Semmler et al. 2007; Dundon et al. 2008), while during submaximal contractions, it has been reported to increase (Semmler et al. 2007; Dartnall et al. 2009) or be unchanged for the first $2 \mathrm{~h}$ after exercise (Piitulainen et al. 2010), and either return to baseline (Semmler et al. 2007; Dartnall et al. 2009) or decrease (Piitulainen et al. 2010) over the next $24 \mathrm{~h}$ in the presence of DOMS. Results for changes in antagonist activity are also inconsistent (Semmler et al. 2007; Turner et al. 2008; Bottas et al. 2009). These differences may result from different protocols used to induce DOMS and to the different severity of impairments induced by the eccentric exercise protocols. As a consequence, the exercise-induced adjustments in neural strategies over a range of force profiles are poorly understood.

Therefore, the aim of the present study was to provide a comprehensive assessment of the effect of eccentric exercise and exercise-induced DOMS on the force profile of the knee extensors and the agonist-antagonist activity during a wide range of force tasks. For this purpose, the subjects performed constant isometric knee extension at target force levels varying between 2.5 and $30 \%$ of maximal force, progressive knee extension to maximal force, and explosive isometric knee extension contractions.

\section{Materials and methods}

\section{Subjects}

Ten healthy subjects (age, mean $\pm \mathrm{SD}, 24.9 \pm 3.2$ years) with no history of musculoskeletal disorders of the lower limbs participated in the study. The subjects were requested to avoid physical exercise and medication during the experimental period. The study was conducted in accordance with the Declaration of Helsinki and approved by the Local Ethics Committee (N-20070019). Subjects provided informed written consent prior to participation in the study.

Experimental procedure

Maximum voluntary contractions (MVC), explosive isometric contraction, and submaximal isometric contraction of the right knee were tested at baseline, immediately following eccentric exercise, and $24 \mathrm{~h}$ after eccentric exercise. During the experimental sessions, pain ratings, force, and EMG parameters were assessed. All measures were performed while the subject was comfortably seated on an isokinetic dynamometer (KinCom Dynamometer, Chattanooga, TN, USA) with their trunk reclined at $15^{\circ}$ in an adjustable chair and their hip and distal thigh firmly strapped to the chair. The rotational axis of the dynamometer was visually aligned with the lateral femoral epicondyle. The right leg was secured to the dynamometer's attachment above the lateral malleolus with the knee in $90^{\circ}$ of flexion. Each subject performed three MVCs of $5 \mathrm{~s}$ each, separated by 2-min rest. Verbal encouragement was given in order to motivate the subject to exceed the previous force level. After $\sim 5 \mathrm{~min}$ rest, the subjects were asked to perform three explosive isometric contractions, 
exerting their maximal force as fast as possible. The trials were separated by 2-min of rest. Verbal encouragement was given in order to ensure concentration and motivation of the subject.

After $15 \mathrm{~min}$ of rest, the subjects performed isometric contractions for $15 \mathrm{~s}$ at target forces of 2.5, 5, 10, 15, 20, and $30 \% \mathrm{MVC}$ in a random order. The submaximal force levels were relative to the MVC measured in each experimental condition (pre-, immediately post- or $24 \mathrm{~h}$ after eccentric exercise). Two minutes of rest was provided between contractions. Subjects were provided with visual feedback of the force exerted, which was displayed on a computer screen of 17 inches in front of them. The isometric tasks required the subject to match the target force level within two error bars of $2 \%$ MVC centered around the target force level. The subjects were encouraged to hold the contraction as steady as possible. For each measurement session, the order of the tasks was: MVC, explosive isometric contractions, and isometric contractions. The post-exercise measurements commenced $\sim 1$ min after the eccentric exercise protocol.

\section{Eccentric exercise}

The eccentric exercise protocol was performed with the KinCom dynamometer (Chattanooga, TN, USA). Subjects performed 4 sets of 25 maximum voluntary eccentric knee extension contractions at a speed of $60^{\circ} \mathrm{s}^{-1}$ between $170^{\circ}$ to $90^{\circ}$ of knee extension $\left(180^{\circ}\right.$ : full knee extension). A rest period of $3 \mathrm{~min}$ was given between each set of contractions. During the exercise, the subject was provided with visual feedback of force and was constantly encouraged to maintain maximal force for each repetition.

\section{Assessment of muscle pain}

To confirm the presence of DOMS $24 \mathrm{~h}$ post-exercise, participants marked their area of pain on a body chart and verbally rated their perceived pain on a scale from 0 ("no soreness") to 10 ("worst soreness imaginable"). The subjects were asked to rate the average pain intensity in the quadriceps during their regular activities of daily living (e.g., descending stairs) since their last visit to the laboratory (over the past $24 \mathrm{~h}$ ).

\section{Surface EMG and force}

For all conditions, knee extension force and surface EMG of the quadriceps and hamstrings muscles were recorded simultaneously. Surface EMG signals were recorded from the vastus medialis (VM), vastus lateralis (VL), biceps femoris (BF), and semitendinosus (ST) muscles using four pairs of surface $\mathrm{Ag}-\mathrm{AgCl}$ electrodes (conductive area 28 mm 2-Ambu Neuroline, Copenhagen, Denmark) with 20-mm inter-electrode distance. After careful preparation of the skin (shaving, abrading, and cleaning with alcohol), the pairs of electrodes were placed over the respective muscles according to published guidelines (Hermens et al. 1999). The position of the electrodes was marked on the skin to assure electrode placement at same location $24 \mathrm{~h}$ after the first session. A reference electrode was placed around the right ankle. Surface EMG signals were amplified as bipolar derivations (EMG amplifier, LISiN-OT Bioelettronica, Torino, Italy), band-pass filtered $(-3 \mathrm{~dB}$ bandwidth, 10-500 Hz), sampled at 2,048 samples/s, and converted to digital data by a 12-bit A/D converter board. Force signals were measured with a load cell (load sensitivity $=0.0048 \mathrm{~V} / \mathrm{N}$ ) connected to the right lower leg and lever arm of the KinCom dynamometer and recorded simultaneously with the EMG.

Data analysis

\section{Force variables}

The reference MVC value corresponded to the maximal force exerted over the three trials. During the explosive contractions, the rate of force development (RFD) and impulse parameters were computed. RFD was computed as the average of the slope of the force-time $(t)$ curve ( $\Delta$ force/ $\Delta$ time) over time intervals of $0-30,0-50,0-100$, and 0-200 ms relative to the onset of the contraction (Aagaard et al. 2002) (Fig. 1). The impulse ( force dt) was determined for the same time intervals. Additionally, maximum RFD ( RFD $\left._{\max }\right)$ was computed as the maximum slope of the force-time curve. The onset of the contraction was defined as the time instant that force exceeded 5 times the standard deviation value observed at baseline.

For each submaximal contraction, the coefficient of variation $(\mathrm{CoV})$ of force was calculated over the entire duration of the contraction. The $\mathrm{CoV}$ of force was computed by dividing the standard deviation (SD) of the force signal by the mean force multiplied by $100[(\mathrm{SD} / \mathrm{mean}$ force)* 100].

\section{EMG variables}

For the reference MVC, the average rectified value (ARV) was computed from the surface EMG signals during a time interval of $250 \mathrm{~ms}$ centered at the time instant of the maximal force. During the explosive contractions, the ARV was calculated for the same time intervals used to calculate the force variables (Fig. 1). ARV was also calculated over two additional time intervals of $50 \mathrm{~ms}$. The first interval was set $70 \mathrm{~ms}$ prior to the onset of the contraction, and the second interval was centered at the time instant of the maximal slope in force (Fig. 1). For the 


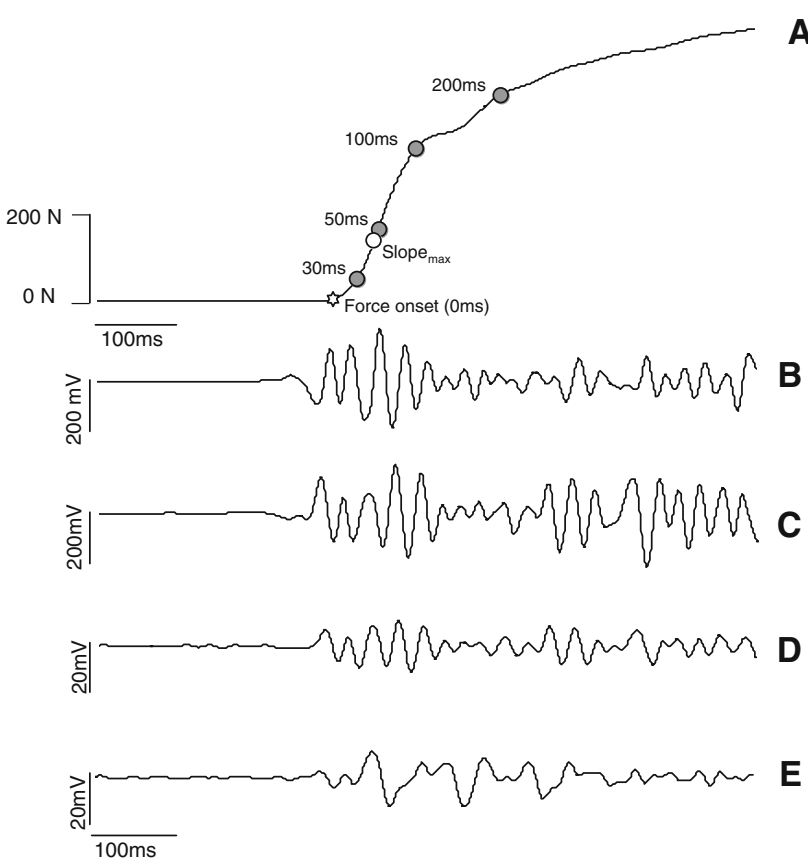

Fig. 1 Representative recordings obtained during explosive isometric knee extensions. a force exerted by the knee extensors. Force onset corresponds to time 0 . Average rate of force development (RFD) and impulse were calculated for the time intervals $0-30,0-50,0-100$, and 0-200 ms. $\mathrm{RFD}_{\max }$ was also calculated from the force-time curve. b surface EMG signals from the vastus medialis. c surface EMG signals from the vastus lateralis. d surface EMG signals from the biceps femoris. e surface EMG signals from the semitendinosus. EMG amplitude of the vasti and hamstrings muscles was computed for the same time intervals aforementioned

submaximal contractions, the ARV was computed from the surface EMG signals in consecutive non-overlapping signals of 1-s epochs and averaged afterward.

\section{Statistical analysis}

The effects of eccentric exercise on the average RFD, impulse and ARV of the vasti, and hamstrings at different time intervals of the explosive contractions were assessed with a three-way repeated measures ANOVA with factors time (baseline, immediately post-, and $24 \mathrm{~h}$ after exercise), time intervals (0-30, 0-50, 0-100, and 0-200 ms), and muscle (VM and VL or BF and ST). During the submaximal contractions, changes in $\mathrm{CoV}$ of the force were assessed with a two-way repeated measures ANOVA with factors time, muscle, and target force levels $(2.5,5,10,15$, 20, and 30\% MVC), while changes in ARV were assessed with a three-way repeated measures ANOVA with factors time, target force levels, and muscle. One-way repeated measures ANOVA with a factor of time was used to assess alterations in $\mathrm{MVC}, \mathrm{RFD}_{\max }$, and $\mathrm{ARV}$ of the vasti muscles at MVC and at the time intervals prior to force onset and centered at the maximum slope of the explosive contractions. Pair-wise comparisons were performed with Student-Newman-Keuls post hoc when ANOVA was significant. Results are reported as means and SD in the text and mean and SE in the figures.

\section{Results}

Pain assessment

Twenty-four hours after exercise, the subjects rated their perceived pain intensity as $4.1 \pm 1.2$ (score out of 10 ).

Maximal voluntary contraction

The MVC was reduced immediately post- $(584.3 \pm 178.2 \mathrm{~N}$, $P<0.01)$ and $24 \mathrm{~h}$ after exercise $(582.3 \pm 202.0 \mathrm{~N}, P<$ $0.001)$ when compared to baseline $(666.1 \pm 207.8 \mathrm{~N})$. Figure 2 illustrates the ARV data from the VM and VL during the MVC. Immediately post-exercise, the ARV of the vasti muscles was reduced by $18.4 \pm 9.2 \%$ when compared to baseline $(P<0.05$; Fig. 2$)$; however, it had recovered $24 \mathrm{~h}$ later. The activity of the BF and ST during the MVC was not significantly affected by the exercise protocol (Fig. 2).

Rate of force development and impulse

$\mathrm{RFD}_{\max }$ decreased progressively across the experimental sessions (main effect time; $P<0.05$ ). When compared to baseline $\left(6,102.9 \pm 2,232.6 \mathrm{~N} \mathrm{~s}^{-1}\right)$, the $\mathrm{RFD}_{\max }$ was significantly reduced immediately post-eccentric exercise $\left(5,273.6 \pm 1,879.5 \mathrm{~N} \mathrm{~s}^{-1} P<0.05\right)$ and remained depressed $24 \mathrm{~h}$ later $\left(5,015.8 \pm 2,049.2 \mathrm{~N} \mathrm{~s}^{-1} P<0.05\right)$. The RFD at different time intervals showed similar behavior (time effect:

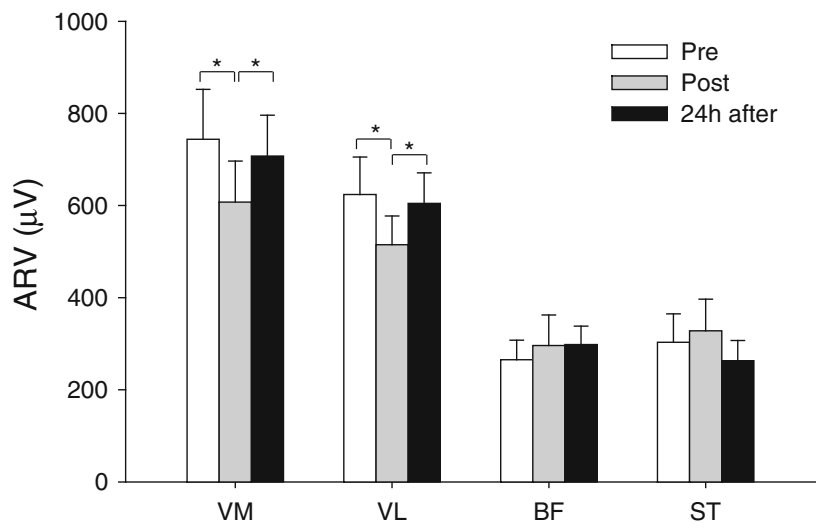

Fig. 2 Mean $\pm \mathrm{SE}$ for the average rectified value (ARV) of the vastus medialis and vastus lateralis obtained during maximal voluntary contraction of the knee extensors. Data were collected at baseline (pre), immediately post-, and $24 \mathrm{~h}$ after eccentric exercise. ${ }^{*} P<0.05$; $* * P<0.01$ 
$P<0.001$, Fig. 3a). A significant interaction between time and interval was found for the impulse $(P<0.001$; Fig. $3 \mathrm{~b})$. During the time intervals 0-100 and 0-200 ms, the impulse was significantly lower immediately post $(-16.9 \pm 16.5 \%$, $P<0.05$ and $-19.6 \pm 18.7 \%, P<0.001$, respectively) and $24 \mathrm{~h}$ after eccentric exercise $(-21.8 \pm 18.5 \%, P<0.05$ and $-23.9 \pm 18.5 \%, P<0.001$, respectively) compared to the baseline condition.

Figure 4 shows the EMG amplitude of the vasti (VM and VL) and the hamstrings muscles (BF and ST) at time intervals prior to the onset of the force (Fig. 4a) and centered around the maximum slope (Fig. 4b).

In both time intervals, the effects of the exercise paradigm on the ARV of the vasti muscles were observed immediately after exercise $(P<0.01$ for both intervals) and during the presence of DOMS ( $P<0.05$ for both intervals). During these time intervals, the vasti ARV values obtained after eccentric exercise represented only $76.4 \pm 32.1 \%$ to $79.6 \pm 28.9 \%$ of the baseline ARV values (Fig. 4). In contrast, during the time interval centered on the maximum slope, the ARV of BF and ST muscles increased immediately post-exercise (147.5 \pm $68.1 \%$; $P<0.01$; Fig. 4b), but returned to baseline values in the following $24 \mathrm{~h}(P<0.01$; Fig. 4b). Similar trends were observed for the ARV at time intervals 0-30, 0-50, 0-100, and 0-200 ms (Table 1).

\section{Submaximal isometric contractions}

Figure 5 illustrates the $\mathrm{CoV}$ of force at the various target force levels measured at baseline, immediately post-exercise, and $24 \mathrm{~h}$ after exercise. A two-way ANOVA showed a significant main effect for both time and load $(P<0.05$ and $P<0.0001$, respectively). The $\mathrm{CoV}$ of force was significantly greater at $2.5,5$, and $10 \%$ MVC (126.7 $\pm 22.6 \%$ to $162.19 \pm 26.9 \%)$ than the CoV observed at 15, 20, and 30\% MVC. The CoV was greater immediately post-exercise (on average $67.5 \pm 46.9 \%$ higher than baseline values, $P<0.05$ ) and remained higher

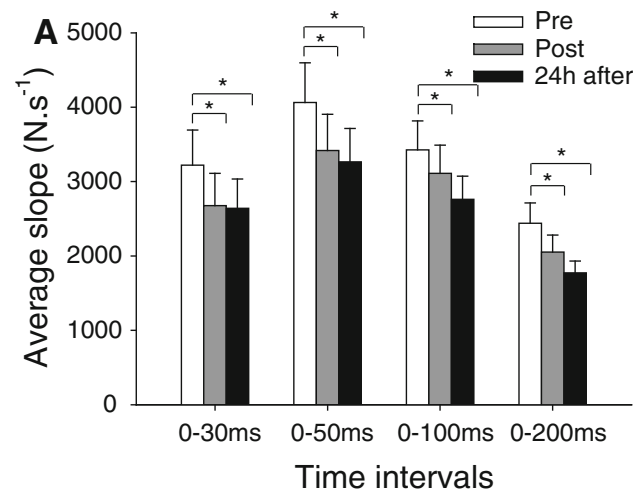

Fig. 3 Mean \pm SE for the average rate of force development (RFD) (a) and impulse (b) obtained during explosive isometric contractions of the knee extensors at time intervals from 0-30, 0-50, 0-100, and
$24 \mathrm{~h}$ after exercise (on average $51.5 \pm 34.5 \%$ higher than pre exercise values, $P<0.05$ ) (Fig. 5). No interaction between time and load was observed $(P=0.32)$.

Changes in force steadiness were accompanied by significant alterations in the ARV of the vasti muscles (interaction between time, load, and muscle, $P<0.05$; Fig. 6) and hamstring muscles (interaction between time and load $P<0.01$; Fig. 7).

At very low loads $(2.5,5$, and $10 \% \mathrm{MVC})$, the ARV of the vasti was not affected immediately post-exercise. However, $24 \mathrm{~h}$ later when DOMS was present, the vasti ARV was greater compared to baseline, except for the VM at $2.5 \%$ MVC (Fig. 6). In contrast, at higher target force levels $(15,20$, and 30\% MVC), the vasti ARV was greater immediately after eccentric exercise, but returned to baseline values $24 \mathrm{~h}$ later (except for the VM at $15 \%$ MVC; Fig. 6). Likewise, the ARV of the ST and BF was greater immediately after eccentric $(P<0.01)$ at the higher force levels $(15,20$, and $30 \% \mathrm{MVC})$, but reduced to baseline values over the following $24 \mathrm{~h}(P<0.001)$ (Fig. 7). In contrast, the ARV of the hamstring muscles was not affected by eccentric exercise at very low loads (2.5, 5, and 10\% MVC) (Fig. 7).

\section{Discussion}

Eccentric exercise and subsequent DOMS are known to affect motor output. However, the effect that eccentric exercise-induced DOMS has on muscle co-activation during different motor tasks is poorly understood. This study examined the effect of eccentric exercise and DOMS on the ability to exert a wide range of force tasks and the effect on the agonist-antagonist activity of the thigh muscles. Force output, assessed either by maximal voluntary contraction, rate of force development, or force steadiness, was impaired both immediately after eccentric exercise and

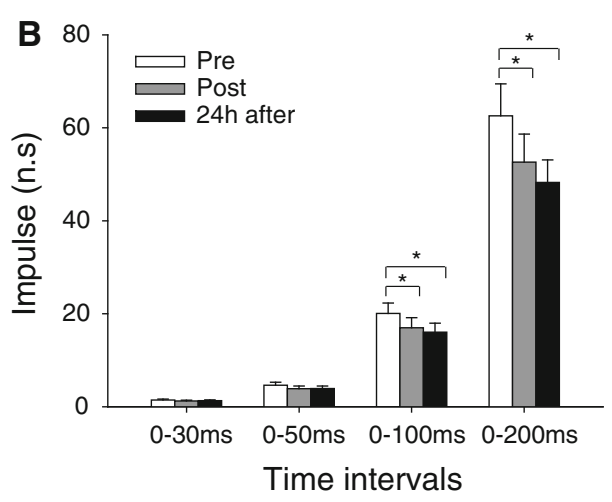

0-200 ms. Data were collected at baseline (pre), immediately post-, and $24 \mathrm{~h}$ after eccentric exercise. $* P<0.05$ 


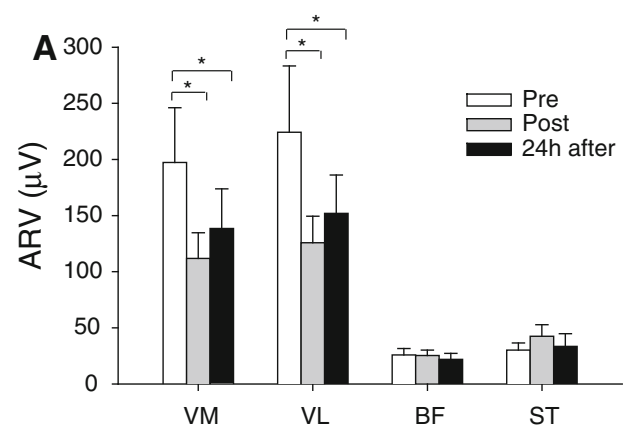

Fig. 4 Mean \pm SE of the EMG average rectified value (ARV) for the vastus medialis (VM), vastus lateralis (VL), biceps femoris (BF), and semitendinosus (ST) obtained during the isometric explosive contractions in all experimental sessions. a ARV calculated in a time

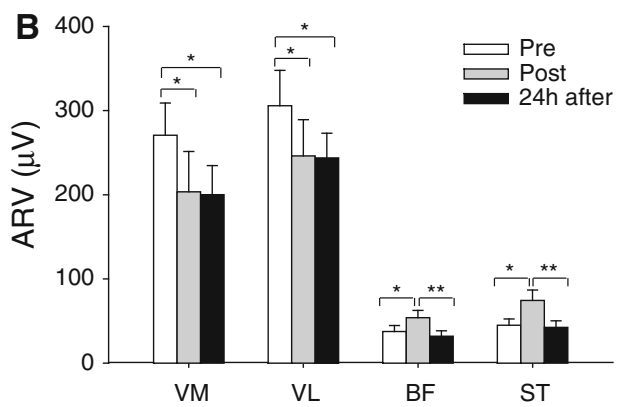

interval $70 \mathrm{~ms}$ prior to the onset of force. b ARV assessed during a time interval of $50 \mathrm{~ms}$ centered at the time instant of the maximum slope. $* P<0.05, * * P<0.01$

Table 1 Average rectified value (ARV) for the vastus medialis (VM), vastus lateralis (VL), biceps femoris (BF), and semitendinosus (ST) obtained during the isometric explosive contractions at time intervals $0-30,0-50,0-100$, and $0-200 \mathrm{~ms}$

\begin{tabular}{|c|c|c|c|c|}
\hline \multirow[t]{2}{*}{ Muscle } & \multicolumn{4}{|l|}{ Average RFD } \\
\hline & $0-30 \mathrm{~ms}$ & $0-50 \mathrm{~ms}$ & $0-100 \mathrm{~ms}$ & $0-200 \mathrm{~ms}$ \\
\hline \multicolumn{5}{|l|}{ Vastus medialis } \\
\hline Baseline & $268.0 \pm 116.9$ & $261.0 \pm 107.9$ & $293.1 \pm 132.7$ & $262.1 \pm 117.5$ \\
\hline Post-exercise & $201.4 \pm 132.0^{\dagger}$ & $190.4 \pm 133.9^{\dagger}$ & $213.6 \pm 160.3^{\dagger}$ & $194.2 \pm 131.6^{\dagger}$ \\
\hline $24 \mathrm{~h}$ after exercise & $215.9 \pm 96.0^{\dagger}$ & $212.6 \pm 96.6^{\dagger}$ & $206.8 \pm 31.7^{\dagger}$ & $182.9 \pm 81.2^{\dagger}$ \\
\hline \multicolumn{5}{|l|}{ Vastus lateralis } \\
\hline Baseline & $300.0 \pm 171.6$ & $281.1 \pm 130.0$ & $352.6 \pm 177.4$ & $327.2 \pm 165.4$ \\
\hline Post-exercise & $219.5 \pm 127.0^{\dagger}$ & $229.2 \pm 127.6^{\dagger}$ & $236.7 \pm 130.3^{\dagger}$ & $227.0 \pm 124.9^{\dagger}$ \\
\hline $24 \mathrm{~h}$ after exercise & $234.3 \pm 104.3^{\dagger}$ & $247.2 \pm 126.1^{\dagger}$ & $281.5 \pm 143.2^{\dagger}$ & $252.8 \pm 123.3^{\dagger}$ \\
\hline \multicolumn{5}{|l|}{ Biceps femoris } \\
\hline Baseline & $42.6 \pm 21.3$ & $40.4 \pm 19.5$ & $46.1 \pm 21.7$ & $41.2 \pm 19.8$ \\
\hline Post-exercise & $73.4 \pm 52.9^{*}$ & $68.3 \pm 43.4^{*}$ & $77.4 \pm 53.5^{*}$ & $69.7 \pm 38.58^{*}$ \\
\hline $24 \mathrm{~h}$ after exercise & $47.1 \pm 39.9$ & $46.8 \pm 30.7$ & $48.4 \pm 38.5$ & $40.7 \pm 26.9$ \\
\hline \multicolumn{5}{|l|}{ Semitendinosus } \\
\hline Baseline & $25.6 \pm 10.7$ & $28.8 \pm 14.1$ & $33.1 \pm 20.6$ & $35.6 \pm 24.6$ \\
\hline Post-exercise & $33.0 \pm 21.0^{*}$ & $33.7 \pm 20.1^{*}$ & $45.9 \pm 25.0^{*}$ & $51.0 \pm 54.6^{*}$ \\
\hline $24 \mathrm{~h}$ after exercise & $23.9 \pm 10.7$ & $26.9 \pm 14.1$ & $33.1 \pm 26.3$ & $29.5 \pm 19.2$ \\
\hline
\end{tabular}

Data were collected at baseline, immediately post-, and $24 \mathrm{~h}$ after eccentric exercise of the quadriceps

$\dagger$ Main effect time $(P<0.01)$. ARV of the VM and VL decreased significantly immediately post-exercise $(P<0.01)$ and remained depressed in the following $24 \mathrm{~h}(P<0.01)$

* Main effect time $(P<0.05)$. ARV of the BF and ST obtained immediately post-exercise was significantly higher than the values observed at baseline $(P<0.05)$ or $24 \mathrm{~h}$ after $(P<0.01)$

$24 \mathrm{~h}$ later during the presence of DOMS. These alterations were accompanied by adjustments in agonist and antagonist muscle activities that followed a different time course depending on the characteristics of the force task.

\section{Muscle soreness}

Subjects reported soreness in the quadriceps muscle $24 \mathrm{~h}$ post-exercise, confirming the presence of DOMS. The average soreness level was approximately 4 out of 10 , which is in agreement with similar studies on the quadriceps (Givoni et al. 2007; Hedayatpour et al. 2010). Although the mechanisms underlying DOMS remain unclear, DOMS is thought to be related to muscle structural damage followed by ion imbalance, inflammation, and pain (Jones et al. 1987; Clarkson et al. 1992; Howell et al. 1993; McBride et al. 2000). The pain is normally triggered by muscle contraction, stretch, pressure, or mechanical stimuli that do not usually induce pain in an unexercised muscle (Proske and Morgan 2001; Taguchi et al. 2005b), making 


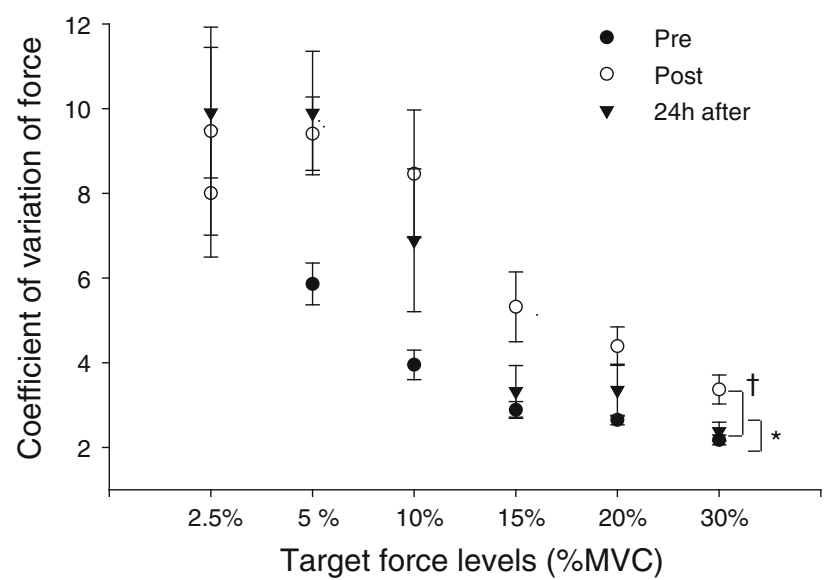

Fig. 5 Mean $\pm \mathrm{SE}$ of the $\mathrm{CoV}$ of the force during submaximal isometric contractions at target force levels of 2.5, 5, 10, 20, and 30\% MVC obtained at baseline (pre), immediately post-, and $24 \mathrm{~h}$ after eccentric exercise. A main effect for time was observed: ${ }^{\dagger} P<0.05$ when comparing baseline to immediate post-exercise, and $* P<0.05$ when comparing baseline to $24 \mathrm{~h}$ after exercise

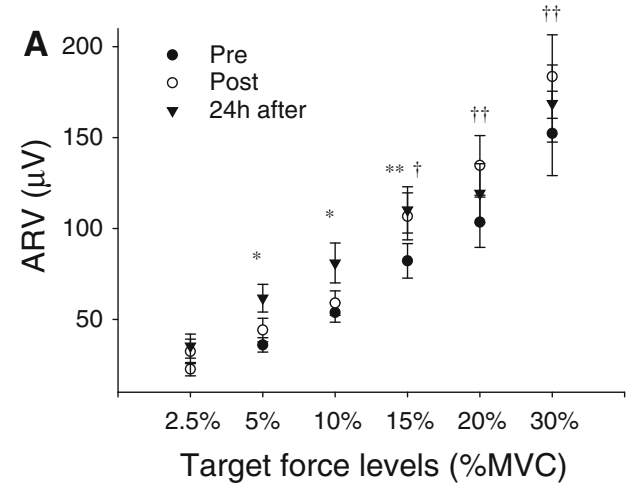

Fig. 6 Mean \pm SE of the EMG average rectified value (ARV) for the a vastus medialis (VM) and b vastus lateralis (VL) during submaximal isometric contractions at target force levels of 2.5, 5, 10, 20, and $30 \%$ MVC obtained at baseline (pre), immediately post-, and $24 \mathrm{~h}$

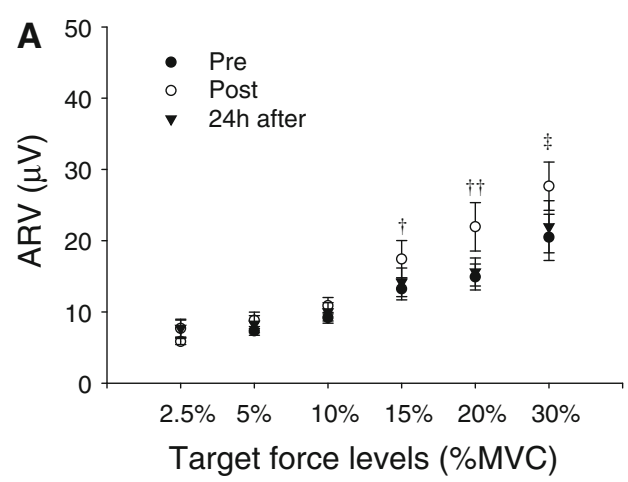

Fig. 7 Mean \pm SE of the EMG average rectified value (ARV) for the a biceps femoris (BF) and $\mathbf{b}$ semitendinosus (ST) during submaximal isometric contractions at target force levels of $2.5,5,10,20$, and $30 \%$ MVC obtained at baseline (pre), immediately post-, and $24 \mathrm{~h}$ after
DOMS a form of hyperalgesia and allodynia (Proske 2005; Taguchi et al. 2005a). Animal studies show that the mechanical sensitivity (threshold and magnitude) of the thin-fiber sensory receptors is facilitated following eccentric exercise (Taguchi et al. 2005a, b). Other studies, however, show that the pain threshold to mechanical pressure in subjects with DOMS increases if larger sensory receptors are blocked, suggesting that changes in the threshold of the larger fiber sensory receptors contributes to DOMS (Barlas et al. 2000; Weerakkody et al. 2003).

Maximal voluntary contractions

Consistent with other studies (Hedayatpour et al. 2007; Semmler et al. 2007), the maximal force of the knee extensors declined immediately post- and $24 \mathrm{~h}$ after eccentric exercise. Assessment of the maximal force during isometric contractions is the most common method of assessing muscle function following eccentric exercise and

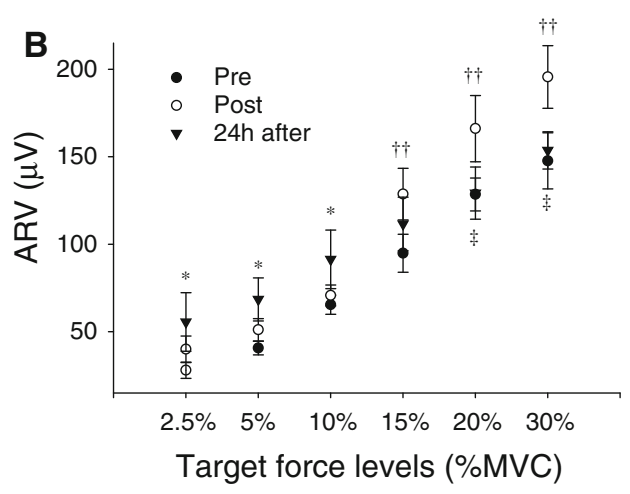

after eccentric exercise. ${ }^{\dagger} P<0.05$ and ${ }^{\dagger} P<0.01$ when comparing baseline to immediate post-exercise. ${ }^{*} P<0.05$ and $* * P<0.01$ when comparing baseline to $24 \mathrm{~h}$ after exercise. ${ }^{\star} P<0.01$ when comparing immediate post- to $24 \mathrm{~h}$ after exercise

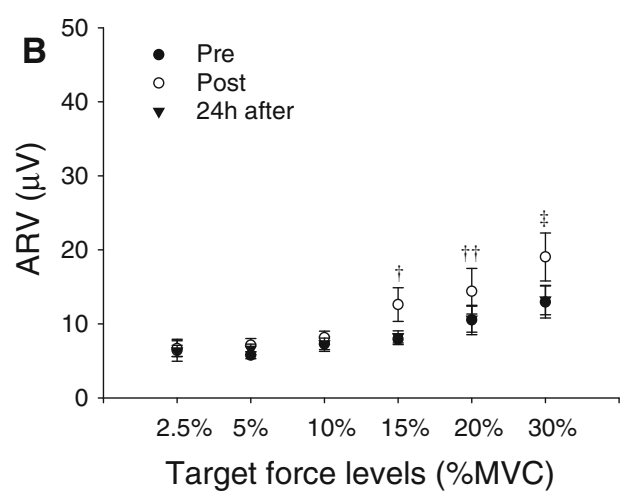

eccentric exercise. ${ }^{\dagger} P<0.01,{ }^{\dagger} P<0.001$, and ${ }^{\star} P<0.001$ when comparing either baseline to immediate post-exercise or immediately post to $24 \mathrm{~h}$ after exercise 
is considered a reliable indicator of muscle damage (Clarkson and Hubal 2002). Eccentric exercise may result in subcellular damage, including Z-line streaming, A-band widening, sarcomere disorganization, and cytoskeletal disruption, which are evident immediately after exercise (Clarkson et al. 1992; Butterfield 2010). Nevertheless, the initial damage may not parallel the early decline in force (Butterfield 2010). This suggests that other alterations, mostly resulting from fatigue, determine the force loss.

In the present study, the reduced EMG amplitude of the vasti observed immediately post-eccentric exercise indicates that peripheral and/or central alterations occurred concurrently with force loss. While voluntary activation (measured with twitch interpolation) of the elbow flexors has been shown to be impaired in the first $24 \mathrm{~h}$ after eccentric exercise, other studies have failed to show such alterations (Pasquet et al. 2000). Peripheral alterations may contribute to the reduced EMG amplitude immediately after eccentric exercise. Animal studies show that eccentric contractions produce marked disturbance of the membrane polarization due to alterations in the extracellular and intracellular $\mathrm{K}^{+}$and $\mathrm{Na}^{+}$concentrations (McBride et al. 2000). A reduction in membrane excitability would reduce the velocity of propagation of the action potentials along the muscle fibers, which partly contributes to reduced force and changes in EMG amplitude. In human studies, a reduction in the conduction velocity of action potentials was observed immediately after eccentric exercise at load intensities from 40 to $100 \%$ MVC and was associated with lower EMG amplitude during maximal voluntary contractions (Piitulainen et al. 2010). Sensitization of group III and IV afferents may also occur following eccentric exercise contributing to inhibition of the motor neuron pool (Bigland-Ritchie et al. 1986; Kaufman et al. 2002) by reducing motor unit discharge rates (Bigland-Ritchie et al. 1986), thus reducing the surface EMG amplitude.

Although the EMG amplitude of the vasti was reduced during the maximal voluntary contraction immediately following eccentric exercise, it had recovered after $24 \mathrm{~h}$, despite a persistent reduction in maximal force and despite the presence of DOMS. Disturbances of excitation-contraction (E-C) coupling have been identified as a major mechanism leading to prolonged force loss after eccentric exercise (for review see Proske and Allen 2005). Furthermore, in the following $24 \mathrm{~h}$, additional muscle damage may occur as a result of a latent effect triggered by several cell signaling cascades (Clarkson et al. 1992; Butterfield 2010). These alterations of the muscle fiber structure and extracellular matrix can contribute to the prolonged impaired force-generating capacity of the muscle (Clarkson et al. 1992; Proske and Allen 2005; Crameri et al. 2007). An additional factor that may contribute to the force loss is a shift of optimum muscle length for peak force. The assessment of maximal force at the same fixed angle preand post-exercise would correspond to different positions on the length-tension curve. However, in the present study, a shift to longer muscle lengths would favor the production of force after eccentric exercise, since the maximal force of the knee extensors is observed at $\sim 70^{\circ}$ of knee flexion (Pincivero et al. 2004), and the fixed angle used in the present study was $90^{\circ}$ of knee flexion.

\section{Explosive isometric contractions}

The rate of force development has a direct influence on the ability to perform rapid movements and is functionally very relevant, for example, it permits the control of unexpected perturbations to maintain postural balance and allows highly trained athletes to reach high movement speeds (Aagaard 2003). Despite the functional importance of this parameter, the effect of unaccustomed eccentric exercise and DOMS on the ability to generate rapid power has received limited attention (Eston et al. 2003). Studies that have been conducted reveal contradictory results; for example, Bottas et al. (2009) reported a poorer deceleration and acceleration of elbow flexion force in the $2 \mathrm{~h}$ following eccentric exercise, with a return to baseline values the day after. On the other hand, Miles et al. (1997) reported a decrease in the peak velocity of the elbow flexors, which remained depressed for several days, and Leger and Milner (2001) did not observe any change in the peak velocity during ballistic metacarpophalangeal abduction after eccentric exercise of the first dorsal interosseus muscle.

In this study, we show that eccentric exercise of the knee extensors significantly reduced the rate of force development in the $24 \mathrm{~h}$ following exercise. Moreover, the impairment of the contractile force characteristics during the explosive contraction was more pronounced than for the MVC $(\sim-19 \%$ vs. $\sim-12 \%$, respectively). This functional alteration was accompanied by significant alterations of the EMG amplitude of the vasti muscles. Contrary to what was observed for the maximal ramp contraction, the explosive contraction was also accompanied by increased antagonist EMG immediately posteccentric exercise.

It is very likely that common mechanisms were involved in the suppression of the maximal and explosive force of the muscle. Nonetheless, some differences in the agonist and antagonist muscle activities were observed, indicating different neural strategies for the two tasks. Contrary to MVC, during the first $200 \mathrm{~ms}$ of the explosive contraction, an increase in the antagonist muscle activity was observed immediately after eccentric exercise. An increase in coactivation of the hamstring muscles would limit the initial acceleration of the muscle contraction by counteracting the agonist muscle action (Crone and Nielsen 1989). This 
effect seems to be a consequence of muscle fatigue since $24 \mathrm{~h}$ later the antagonist muscle activity was restored to normal values. Despite this, the rapid contractile properties of the agonist remained depressed $24 \mathrm{~h}$ later when DOMS was present, indicating that other mechanisms are involved in such impairment.

Immediately post-eccentric exercise, the EMG amplitude of the vasti muscles was depressed more for the explosive contraction than for the maximal isometric contraction $(\sim-24 \%$ vs. $\sim-18$ compared to baseline values, respectively) and remained depressed for the following $24 \mathrm{~h}$, but only for the explosive contraction. Furthermore, a pronounced suppression of the vasti EMG amplitude was also evident in the time interval prior to force onset without any alterations of antagonist muscle activity. This indicates that the reduced capacity to generate rapid muscle contractions is partly due to neural impairments that would not manifest during a progressive increase of the maximal force. Desmedt and Godaux (1977) reported distinct differences in the pattern of motor unit recruitment in voluntary ramp contractions compared to ballistic contractions. For instance, a rapid increment of motor unit discharge rates and a high incidence of discharge doublets in the very early phase of a rapid contraction play a crucial role in the rate of force development (Desmedt and Godaux 1977; Van Cutsem et al. 1998). Thus, a possible explanation for concurrent suppression of the rapid contractile properties and EMG amplitude would be a reduction in the maximal motor unit discharge rates and/or incidence of discharge doublets in the early phase of the explosive contraction. This limitation may arise from impairments in central motor commands. Ballistic contractions are completed in a very short time, which implies that central motor commands need to be pre-programmed and adjusted according to the expected force requirements.

\section{Submaximal isometric tasks}

Force steadiness was reduced immediately after eccentric exercise and remained reduced $24 \mathrm{~h}$ after exercise when DOMS was present, especially at very low loads. This study shows that these alterations were accompanied by different alterations of agonist and antagonist muscle activities, depending on the target force level.

The origin of increased force fluctuation is dependent on the interaction of multiple features of motor unit behavior, which change as a function of the contraction intensity (Taylor et al. 2003). Alterations in recruitment and ratecoding properties of the motor units and activation pattern of the motor unit population (e.g., motor unit synchronization and coherence) would affect force variability. Studies have shown that eccentric exercise may reduce motor unit recruitment thresholds (Dartnall et al. 2009) and increase the minimum discharge rate variability (Dartnall et al. 2009) and the motor unit coherence and motor unit synchronization (Dartnall et al. 2008). Moreover, these alterations remain for $24 \mathrm{~h}$ after eccentric exercise when DOMS was present (Dartnall et al. 2008, 2009) and are associated with increased force fluctuations. These effects are more pronounced at low force levels since the relative contribution of each newly recruited motor unit to the net force is greatest at low forces since there are a smaller number of active motor units (Semmler et al. 2007). These mechanisms may alter the EMG amplitude due to the summation of the action potentials at the muscle surface (Farina et al. 2004).

Previous studies (Semmler et al. 2007; Dartnall et al. 2008) have shown that the largest change in EMG amplitude of the biceps brachii occurs at low contraction levels (between 5 and 20\% MVC) immediately post-eccentric exercise of the elbow flexors, and by $24 \mathrm{~h}$, these values return to baseline (Semmler et al. 2007; Dartnall et al. 2008). However, Semmeler et al. showed the greatest change in agonist activity at 5\% MVC, whereas Dartnall et al. (2008) observed the greatest change at $20 \%$ MVC with no significant alteration of the EMG amplitude occurring at lower forces. In the present study, the EMG amplitude of the vasti muscles increased immediately after eccentric exercise, but only at force levels greater than $15 \%$ MVC and was accompanied by increased activation of the antagonist muscles. The vasti and hamstring EMG amplitude returned to baseline values $24 \mathrm{~h}$ post-exercise when DOMS was present for contractions at $15 \%$ and above, while at the very low force levels (2.5-10\%), an increase in the vasti EMG amplitude was observed. These results suggest that the neural adjustments among the agonist and antagonist muscles follow a different time course and are dependent on the force level, which may help explain the variability of results reported in the literature.

The delayed increase in the EMG amplitude for the vasti at very low force levels may result from an excessive loss of force generated at low frequencies (Jones 1996). Dundon et al. (2008) showed that the recovery of low-frequency fatigue following eccentric exercise of the elbow flexors was significantly prolonged (up to $24 \mathrm{~h}$ ), and therefore present during DOMS, with the most prominent changes occurring $2 \mathrm{~h}$ after exercise. Moreover, the variations in the EMG amplitude were explained by low-frequency fatigue, with the strongest associations occurring between 5 and 40\% MVC (Dundon et al. 2008). Lowfrequency fatigue has been associated with impairments in E-C coupling, mainly due to reduction in the release of $\mathrm{Ca}^{2+}$ from the sarcoplasmic reticulum (Bruton et al. 1998). This phenomenon is more pronounced after eccentric than after concentric or isometric exercise, probably due to muscle damage induced by lengthening contractions (Allen et al. 1995). 
Increased antagonist activity has been observed $2 \mathrm{~h}$ following eccentric exercise of the elbow flexors, but returned to baseline values 24 h later (Semmler et al. 2007; Dundon et al. 2008). In these studies, the increased antagonist activity was observed during sustained contractions with loads ranging between 5 and 60\% MVC. In our study, increased antagonist activity was only observed at loads up to $15 \%$ MVC, while no changes were observed for very low loads. The differences between results might be related with the type of experimental protocol and/or differences in the neural control strategies of the upper versus lower limb. Increased antagonist muscle activity may represent a mechanism to increase movement precision (Crone and Nielsen 1989) and may be more relevant for the upper than lower limbs.

\section{Methodological considerations}

Measurements of the absolute EMG amplitude, as used in this study, are influenced by many factors, even when the positions of the electrodes are marked between sessions; for example, reduced EMG amplitude after eccentric exercise could be due to local inflammation within the muscle, or a decline in neuromuscular transmission (Piitulainen et al. 2008). This issue could be avoided by normalizing the EMG amplitude; however, there is no consensus on the most robust normalization method; for example, normalizing with respect to the maximal M-wave is limited by the different influence that peripheral factors have on the M-wave amplitude with respect to the voluntary EMG amplitude (Keenan et al. 2006). In the absence of normalization, the EMG amplitude is variable from session to session; however, its repeatability has been quantified in previous studies (e.g., Rainoldi et al. 2001) and is sufficient for identifying statistically significant differences as in this study.

This study investigated the immediate and delayed effects of eccentric exercise of the quadriceps on agonist-antagonist activity during a range of motor tasks. These findings may not be applicable to other muscle groups, since different muscle groups can be more or less susceptible to muscle damage from eccentric exercise (Chen et al. 2011). Although the immediate effects of eccentric exercise were assessed, these measurements would have been influenced by the acute effects of fatigue. Additional measurements performed 1 or $2 \mathrm{~h}$ post-exercise would have allowed the effects of eccentric exercise to be assessed more specifically.

\section{Conclusion}

The present study showed that all examined force output profiles (maximal voluntary contraction, rate of force development, and force steadiness of the knee extensors) were disturbed immediately following eccentric exercise of the knee extensors and persisted for $24 \mathrm{~h}$ after exercise when DOMS was present. These alterations were accompanied by different adjustments of agonist and antagonist muscle activities. Moreover, the neural adjustments followed a different time course depending on the characteristics of the force task. These compensatory mechanisms may increase the risk of further injury if a premature return to sport or exercise is attempted.

Acknowledgments This work was supported by the Fundação para a Ciência e Tecnologia (FCT) of Portugal (PhD Grant ID-SFRH/BD/ $31796 / 2006$ to CVC).

Open Access This article is distributed under the terms of the Creative Commons Attribution Noncommercial License which permits any noncommercial use, distribution, and reproduction in any medium, provided the original author(s) and source are credited.

\section{References}

Aagaard P (2003) Training-induced changes in neural function. Exerc Sport Sci Rev 31:61-67

Aagaard P, Simonsen EB, Andersen JL, Magnusson P, Dyhre-Poulsen $P$ (2002) Increased rate of force development and neural drive of human skeletal muscle following resistance training. J Appl Physiol 93(4):1318-1326

Allen DG, Westerblad H, Lannergren J (1995) The role of intracellular acidosis in muscle fatigue. Adv Exp Med Biol 384:57-68

Barlas P, Craig JA, Robinson J, Walsh DM, Baxter GD, Allen JM (2000) Managing delayed-onset muscle soreness: lack of effect of selected oral systemic analgesics. Arch Phys Med Rehabil 81(7):966-972

Bawa P (2002) Neural control of motor output: can training change it? Exerc Sport Sci Rev 30(2):59-63

Bigland-Ritchie BR, Dawson NJ, Johansson RS, Lippold OC (1986) Reflex origin for the slowing of motoneurone firing rates in fatigue of human voluntary contractions. J Physiol 379:451-459

Bottas R, Nicol C, Komi PV, Linnamo V (2009) Adaptive changes in motor control of rhythmic movement after maximal eccentric actions. J Electromyogr Kinesiol 19:347-356

Bruton JD, Lannergren J, Westerblad H (1998) Mechanisms underlying the slow recovery of force after fatigue: importance of intracellular calcium. Acta Physiol Scand 162(3):285-293

Butterfield TA (2010) Eccentric exercise in vivo: strain-induced muscle damage and adaptation in a stable system. Exerc Sport Sci Rev 38(2):51-60

Chen TC, Lin KY, Chen HL, Lin MJ, Nosaka K (2011) Comparison in eccentric exercise-induced muscle damage among four limb muscles. Eur J Appl Physiol 111(2):211-223

Clarkson PM, Hubal MJ (2002) Exercise-induced muscle damage in humans. Am J Phys Med Rehabil 81(11 Suppl):S52-S69

Clarkson PM, Nosaka K, Braun B (1992) Muscle function after exercise-induced muscle damage and rapid adaptation. Med Sci Sports Exerc 24(5):512-520

Crameri RM, Aagaard P, Qvortrup K, Langberg H, Olesen J, Kjaer M (2007) Myofibre damage in human skeletal muscle: effects of electrical stimulation versus voluntary contraction. J Physiol 583(Pt 1):365-380 
Crone C, Nielsen J (1989) Spinal mechanisms in man contributing to reciprocal inhibition during voluntary dorsiflexion of the foot. J Physiol 416:255-272

Dartnall TJ, Nordstrom MA, Semmler JG (2008) Motor unit synchronization is increased in biceps brachii after exerciseinduced damage to elbow flexor muscles. J Neurophysiol 99:1008-1019

Dartnall TJ, Rogasch NC, Nordstrom MA, Semmler JG (2009) Eccentric muscle damage has variable effects on motor unit recruitment thresholds and discharge patterns in elbow flexor muscles. J Neurophysiol 102(1):413-423

Desmedt JE, Godaux E (1977) Ballistic contractions in man: characteristic recruitment pattern of single motor units of the tibialis anterior muscle. J Physiol 264(3):673-693

Dundon JM, Cirillo J, Semmler JG (2008) Low-frequency fatigue and neuromuscular performance after exercise-induced damage to elbow flexor muscles. J Appl Physiol 105:1146-1155

Enoka RM (1988) Muscle strength and its development. New perspectives. Sports Med 6(3):146-168

Eston RG, Byrne C, Twist C (2003) Muscle function after exerciseinduced muscle damage: Considerations for atheletic perfomance in children and adults. J Exerc Sci Fit 1:85-96

Farina D, Merletti R, Enoka RM (2004) The extraction of neural strategies from the surface EMG. J Appl Physiol 96:1486-1495

Givoni NJ, Pham T, Allen TJ, Proske U (2007) The effect of quadriceps muscle fatigue on position matching at the knee. J Physiol 584(Pt 1):111-119

Hedayatpour N, Arendt-Nielsen L, Farina D (2007) Motor unit conduction velocity during sustained contraction of the vastus medialis muscle. Exp Brain Res 180(3):509-516

Hedayatpour N, Falla D, Arendt-Nielsen L, Farina D (2010) Effect of delayed-onset muscle soreness on muscle recovery after a fatiguing isometric contraction. Scand J Med Sci Sports 20(1):145-153

Hermens H, Freriks B, Merletti R, Stegeman DF, Blok J, Rau G, Disselhorst-Klug C, Hagg G (1999) European recommendations for surface electromyography. Results of the SENIAM project. Roessingh Research and Development

Howell JN, Chleboun G, Conatser R (1993) Muscle stiffness, strength loss, swelling and soreness following exercise-induced injury in humans. J Physiol 464:183-196

Jones DA (1996) High-and low-frequency fatigue revisited. Acta Physiol Scand 156(3):265-270

Jones DA, Newham DJ, Clarkson PM (1987) Skeletal muscle stiffness and pain following eccentric exercise of the elbow flexors. Pain 30(2):233-242

Kaufman MP, Hayes SG, Adreani CM, Pickar JG (2002) Discharge properties of group III and IV muscle afferents. Adv Exp Med Biol 508:25-32

Keenan KG, Farina D, Merletti R, Enoka RM (2006) Influence of motor unit properties on the size of the simulated evoked surface EMG potential. Exp Brain Res 169(1):37-49

Leger AB, Milner TE (2001) Motor impairment in the human hand following eccentric exercise. Eur J Appl Physiol 84:213-220

McBride TA, Stockert BW, Gorin FA, Carlsen RC (2000) Stretchactivated ion channels contribute to membrane depolarization after eccentric contractions. J Appl Physiol 88(1):91-101

Meszaros AJ, Iguchi M, Chang SH, Shields RK (2010) Repetitive eccentric muscle contractions increase torque unsteadiness in the human triceps brachii. J Electromyogr Kinesiol 20(4):619-626

Miles MP, Ives JC, Vincent KR (1997) Neuromuscular control following maximal eccentric exercise. Eur J Appl Physiol Occup Physiol 76:368-374

Paschalis V, Giakas G, Baltzopoulos V, Jamurtas AZ, Theoharis V, Kotzamanidis C, Koutedakis Y (2007) The effects of muscle damage following eccentric exercise on gait biomechanics. Gait Posture 25:236-242
Pasquet B, Carpentier A, Duchateau J, Hainaut K (2000) Muscle fatigue during concentric and eccentric contractions. Muscle Nerve 23(11):1727-1735

Piitulainen H, Komi P, Linnamo V, Avela J (2008) Sarcolemmal excitability as investigated with M-waves after eccentric exercise in humans. J Electromyogr Kinesiol 18:672-681

Piitulainen H, Bottas R, Komi P, Linnamo V, Avela J (2010) Impaired action potential conduction at high force levels after eccentric exercise. J Electromyogr Kinesiol 20(5):879-887

Pincivero DM, Salfetnikov Y, Campy RM, Coelho AJ (2004) Angleand gender-specific quadriceps femoris muscle recruitment and knee extensor torque. J Biomech 37(11):1689-1697

Prasartwuth O, Taylor JL, Gandevia SC (2005) Maximal force, voluntary activation and muscle soreness after eccentric damage to human elbow flexor muscles. J Physiol 567(Pt 1):337-348

Proske U (2005) Muscle tenderness from exercise: mechanisms? J Physiol 564(Pt 1):1

Proske U, Allen TJ (2005) Damage to skeletal muscle from eccentric exercise. Exerc Sport Sci Rev 33(2):98-104

Proske U, Morgan DL (2001) Muscle damage from eccentric exercise: mechanism, mechanical signs, adaptation and clinical applications. J Physiol 537(Pt 2):333-345

Rainoldi A, Bullock-Saxton JE, Cavarretta F, Hogan N (2001) Repeatability of maximal voluntary force and of surface EMG variables during voluntary isometric contraction of quadriceps muscles in healthy subjects. J Electromyogr Kinesiol 11(6):425-438

Sargeant AJ, Dolan P (1987) Human muscle function following prolonged eccentric exercise. Eur J Appl Physiol Occup Physiol 56(6):704-711

Saxton JM, Clarkson PM, James R, Miles M, Westerfer M, Clark S, Donnelly AE (1995) Neuromuscular dysfunction following eccentric exercise. Med Sci Sports Exerc 27(8):1185-1193

Semmler JG, Tucker KJ, Allen TJ, Proske U (2007) Eccentric exercise increases EMG amplitude and force fluctuations during submaximal contractions of elbow flexor muscles. J Appl Physiol 103(3):979-989

Taguchi T, Matsuda T, Tamura R, Sato J, Mizumura K (2005a) Muscular mechanical hyperalgesia revealed by behavioural pain test and c-Fos expression in the spinal dorsal horn after eccentric contraction in rats. J Physiol 564(Pt 1):259-268

Taguchi T, Sato J, Mizumura K (2005b) Augmented mechanical response of muscle thin-fiber sensory receptors recorded from rat muscle-nerve preparations in vitro after eccentric contraction. J Neurophysiol 94(4):2822-2831

Taylor AM, Christou EA, Enoka RM (2003) Multiple features of motor-unit activity influence force fluctuations during isometric contractions. J Neurophysiol 90(2):1350-1361

Tracy BL, Maluf KS, Stephenson JL, Hunter SK, Enoka RM (2005) Variability of motor unit discharge and force fluctuations across a range of muscle forces in older adults. Muscle Nerve 32:533-540

Turner TS, Tucker KJ, Rogasch NC, Semmler JG (2008) Impaired neuromuscular function during isometric, shortening, and lengthening contractions after exercise-induced damage to elbow flexor muscles. J Appl Physiol 105:502-509

Van Cutsem M, Duchateau J, Hainaut K (1998) Changes in single motor unit behaviour contribute to the increase in contraction speed after dynamic training in humans. J Physiol 513(Pt 1):295-305

Weerakkody NS, Percival P, Hickey MW, Morgan DL, Gregory JE, Canny BJ, Proske U (2003) Effects of local pressure and vibration on muscle pain from eccentric exercise and hypertonic saline. Pain 105(3):425-435

Yu JG, Thornell LE (2002) Desmin and actin alterations in human muscles affected by delayed onset muscle soreness: a high resolution immunocytochemical study. Histochem Cell Biol 118(2):171-179 